\title{
Contour Based Path Planning with B-Spline Trajectory Generation for Unmanned Aerial Vehicles (UAVs) over Hostile Terrain
}

\author{
Ee-May Kan ${ }^{1}$, Meng-Hiot Lim ${ }^{1}$, Swee-Ping $\mathrm{Yeo}^{2}$, Jiun-Sien $\mathrm{Ho}^{3}$, Zhenhai Shao ${ }^{4}$ \\ ${ }^{1}$ Nanyang Technological University, Singapore City, Singapore; ${ }^{2}$ National University of Singapore, Singapore City, Singapore;
${ }^{3}$ Temasek Polytechnic, Singapore City, Singapore; ${ }^{4}$ University of Electronic Science Technology of China, Chengdu, China.
Email: ka0001ay@e.ntu.edu.sg, emhlim@ntu.edu.sg, eleyeosp@nus.edu.sg, jiunsien@tp.edu.sg
}

Received May $20^{\text {th }}, 2011$; revised July $1^{\text {st }}, 2011$; accepted July $8^{\text {th }}, 2011$.

\begin{abstract}
This research focuses on trajectory generation algorithms that take into account the stealthiness of autonomous UAVs; generating stealthy paths through a region laden with enemy radars. The algorithm is employed to estimate the risk cost of the navigational space and generate an optimized path based on the user-specified threshold altitude value. Thus the generated path is represented with a set of low-radar risk waypoints being the coordinates of its control points. The radar-aware path planner is then approximated using cubic B-splines by considering the least radar risk to the destination. Simulated results are presented, illustrating the potential benefits of such algorithms.
\end{abstract}

Keywords: Unmanned Aerial Vehicles (UAVs), Radar, Path Planning, B-Splines

\section{Introduction}

Unmanned aerial vehicles (UAVs) are increasingly being used in real-world applications [1-3]. They are typically surveillance and reconnaissance vehicles operated remotely by a human operator from a ground control station; they have no on-board guidance capabilities that give them some level of autonomy, for example, to re-plan a trajectory in the event of a change in the environment or mission. With such rudimentary capabilities, only simple tasks can be accomplished and the operation is also limited to simple or uncomplicated situations, typically in well-characterized environments. It is useful to endow UAVs with more advanced guidance capabilities, in particular capabilities that increase the vehicle's autonomy to allow for more complex missions or tasks. Currently operating UAVs use rudimentary guidance technologies, such as following pre-planned or manually provided waypoints. Over the years, advances in software and computing technology have fuelled the development of a variety of new guidance methodologies for UAVs. The availability of various guidance technologies and understanding of operational scenarios create opportunities towards refining and implementing such advanced guidance concepts. The basic difficulties include partially known and changing environment, extraneous factors, such as threats, evolving mission elements, tight timing and positioning requirements. Moreover, these must be tackled, while at the same time, explicitly accounting for the actual vehicle manoeuvring capabilities, including dynamics and flight-envelope constraints. The term flight envelope refers to the parameters within which an aerial vehicle may be safely flown under varying, though expected, wind speed, wing loading, wind shear, visibility and other flight conditions without resorting to extreme control measures such as abnormal spin, or stall recovery, or crash landing. These aerial vehicles are usually mobilized to carry out critical missions in high-risk environments, particularly in situations where it may be hazardous for human operators. However, such missions demand a high level of stealth, which has a direct implication on the safety and success of the mission. Therefore it is important to minimize the risk of detection or more specifically, the probability of detection of the UAVs by enemy radars.

There has been extensive research in the area of path planning especially in the artificial intelligence, and optimization with most restricted to two-dimensional (2D) paths $[4,5]$. Different conventional approaches have been developed to solve the path planning problem, such as the cell decomposition, Djikstra's algorithm, road map and potential field [6-9]. Sleumer and Tschichold [6] present 
an algorithm for the automatic generation of a map that describes the operation environment of a building consisting of line segments, representing the walls as an input. The algorithm is based on the exact cell decomposition approach and generates a connectivity graph based on cell indices. However, for successful implementation of exact cell decomposition, it is required that the geometry of each cell be simple. Moreover, it is important to test the adjacency of two cells to find a path crossing the portion of the boundary shared by the two cells. Both exact cell decomposition and approximate cell decomposition methods are accurate, yet may be computationally expensive when used in a terrain environment since numerous obstacles at varying elevations could be found. They are only effective when the environment contains a countable number of obstacles.

Another technique used to effectively represent the alternate paths generated by a path planner is to use Bsplines. B-splines allow a parametric curved path to be described by only a few control points rather than the entire curve segmented which could be as many as thousands of sections, depending on the length and curvature of the path. Recent methods include modelling the trajectory using splines based on elastic band theory $[10,11]$, and interactive manipulation of control points for spline trajectory generation $[12,13]$. A series of cubic splines was employed in [14] to connect the straight line segments in a near-optimal manner, whereas the algorithm presented in [15] yields extremal trajectories that transition between straight-line path segments smoothly in a time-optimal fashion.

Previous efforts $[16,17]$ have concentrated on path planning over a hostile radar zones based on the amount of energy received by enemy radar at several locations. In this paper, we investigate on the number and placement of control points within a hostile region by considering the complexity of the terrain. We take into account the path optimality within a hostile region by considering the complexity of the terrain. We propose an efficient algorithm to identify the waypoints based on the topographic information of the enemy terrain. The waypoint generation process is based on the user-specified threshold flying altitude. The threshold altitude to avoid radar detection and terrain collisions is determined based on the knowledge of the enemy terrain. The navigational path between the waypoints is considered when minimizing the exposure to a radar source. Additionally, the generated trajectory is of minimal length, subject to the stealthy constraint and satisfies the aircraft's dynamic constraints.

Details on the formulation of the problem are presented in this paper. We model this problem as described in the following section and adopt a heuristic-based approach to solve it. The rest of this paper is organized as follows: Section 2 gives a description of the problem model used for measuring stealth in this work and illustrates the calculation of the radar risks. Section 3 demonstrates the details of the problem formulation and Section 4 presents the solution approach of the route planner, substantiated with simulated results. The conclusion and future work are presented in Section 5.

\section{Modelling}

In this section, the model we used throughout this work is presented. The integrated model of the UAV and radar has the following features. The Radar Cross Section (RCS) of the UAV depends on both the aspect and bank angles whereas the turn rate of the UAV is determined by its bank angle. Hence, the RCS and aircraft dynamics are coupled through the aspect and bank angles.

\subsection{Measuring Stealth Based on Aircraft Model}

The bank-to-turn aircraft is assumed to move in a horizontal plane at a constant altitude according to the equations [18],

$$
x=v \cos \varphi, \quad y=v \sin \varphi, \quad \varphi=\frac{u}{v},|u|<U
$$

where $x$ and $y$ are the Cartesian coordinates of the aircraft, $\varphi$ is the heading angle as shown in Figure 1, $v$ is the constant speed, $u$ is the input signal and the acceleration is normal to the flight path vector, followed by $U$ is the maximum allowable lateral acceleration. Figures 2 and 3 show the dependence of RCS on aspect and bank angles.

Let

$$
\begin{gathered}
\theta=\arctan \left(\frac{y}{x}\right), \lambda=\theta-\varphi+\pi, \\
\phi=\arctan \left(\frac{z}{\sqrt{x^{2}+y^{2}}}\right)
\end{gathered}
$$

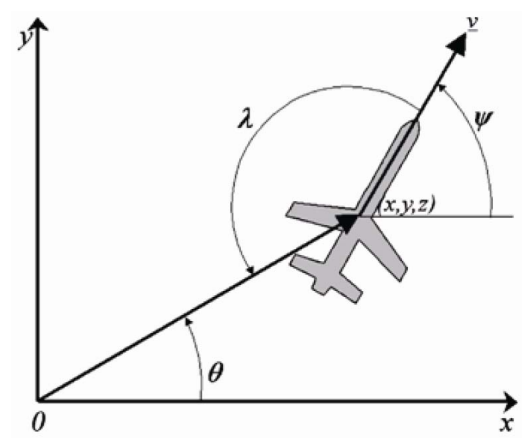

Figure 1. Aircraft position, velocity, azimuth, heading, and aspect angles. 


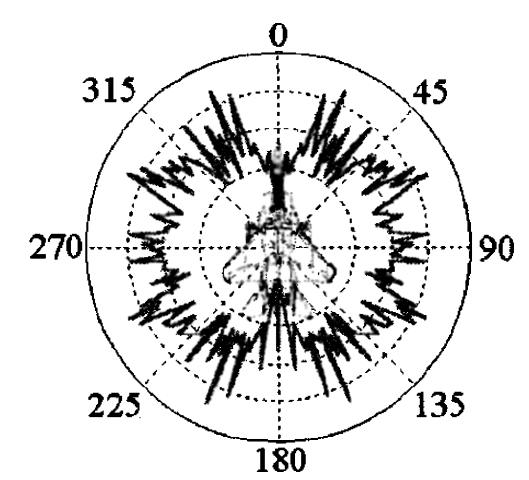

Figure 2. RCS as a function of aspect angle.

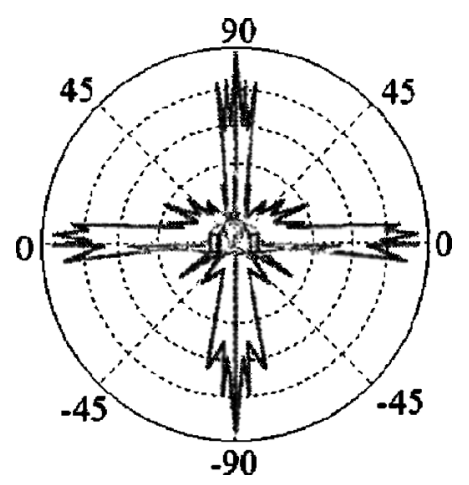

Figure 3. RCS as a function of bank angle.

be the azimuth, aspect, and elevation angles, respectively, where $z$ is the aircraft altitude. Let the bank angle $\mu$ be given by

$$
u=\arctan \left(\frac{u}{g}\right)
$$

where $g$ is the acceleration of gravity. We model the RCS of the aircraft as function of the aspect angle $\lambda$, the elevation angle $\varphi$, and the bank angle $\mu$, so that

$$
\mathrm{RCS}=\mathrm{RCS}=\sigma(\lambda, \varphi, \mu)
$$

The RCS obtained from Equation (4) is used as an estimate value in Equation (6) for measuring stealth in this work. As an example, real aircraft RCS measurements as functions of aspect and bank angles are shown in Figures 3 and 4 [18]. The following section illustrates the calculation of the cost associated to radar risk that will be undertaken by the UAV.

\subsection{Radar Model}

The radar model in this work will be presented in terms of its inputs (aircraft range and RCS) and output (an estimate of the probability that an aircraft can be tracked for an interval of time). For the sake of simplicity, we assume that the radar is located at $(x, y, z)$ of the navigational space. Let

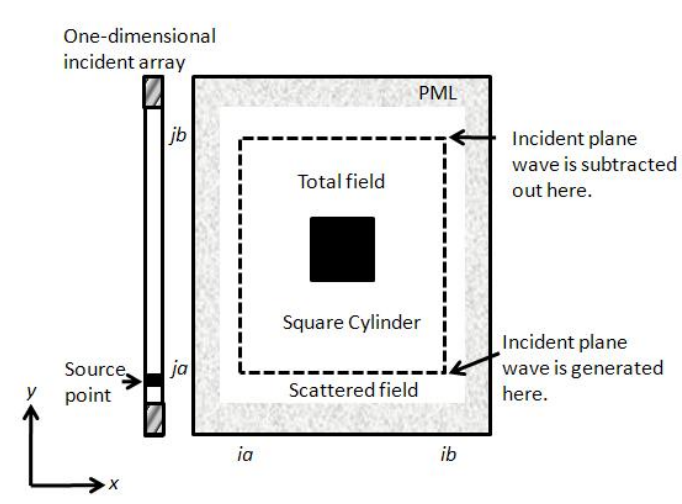

Figure 4. Square cylinder for RCS modelling.

$$
R=\sqrt{(\Delta x)^{2}+(\Delta y)^{2}+(\Delta z)^{2}}
$$

be the aircraft range from the enemy radar to the aircraft. The radar detects the aircraft by receiving a sequence of radio frequency pulses reflected from it at fixed observation times. To generate a stealthy path for the UAV, we have to take into account the energy reflected to enemy radar site as the enemy radar tracks the location, speed, and direction of an aircraft. The range at which radar can detect an object is related to the power transmitted by the radar, the fidelity of the radar antenna, the wavelength of the radar signal, and the RCS of the aircraft [19]. The RCS is the area a target would have to occupy to produce the amount of reflected power that is detected back at the radar. RCS is integral to the development of radar stealth technology, particularly in applications involving aircraft. For example, a stealth aircraft which is designed to be undetectable will have design features that give it a low RCS. Low RCS offers advantages in detection range reduction as well as increasing the effectiveness of decoys against radar-seeking threats. The size of a target's image on radar is measured by the RCS and denoted by the symbol $\sigma$ and expressed in square meters. The azimuth and range detected by the radar serve as the inputs to a tracking system, typically based on one or more Kalman filters. The purpose of the tracking system is to provide a predicted aircraft position and velocity so that a decision can be made to launch a missile and guide it to intercept. RCS modelling and reduction is crucial in designing stealth weapon systems. We make use of FiniteDifference Time-Domain (FDTD) Method technique in RCS modelling. The numerical technique is general, geometry-free, and can be used arbitrarily for any target, within the limitations of computer memory and speed. Figure 4 shows the geometric surface for the RCS simulation with the FDTD algorithm. The FDTD-based package was supplied in [20] for the RCS prediction of 
various simple canonical targets. The complete RCS signature of a target is described by the frequency response of the target illuminated by plane waves from all possible physical angles. Realistic targets are frequently very large and are often largely made up of highly conductive materials.

The FDTD-based RCS prediction procedure is as follows:

- The target, located in the computational space, is illuminated by a Gaussian-pulse plane wave from a given direction;

- The scattered fields are simulated inside the computational volume until all transients dissipate;

- The time-domain far fields in the spherical coordinates are then extrapolated by using equivalent current via the near-field to far-field routine, over a closed, virtual surface surrounding the target.

For either vertical or horizontal planes, co-polarized or cross-polarized RCS behaviour is computed after the simulation for a given frequency range. Figure 5 illustrates the RCS behaviour with respect to the azimuth angle based on the RCS modelling described above.

Subsequently the computation of the energy received by a radar is given by the radar range equation [21]

$$
S \cong \frac{P_{\text {avg }} G \sigma t_{o t} A_{e}}{\left(4 \pi^{2}\right) R^{4}}
$$

where $\mathrm{S}$ is the signal energy received by the radar, $P_{\text {avg }}$ is the average power transmitted by the radar, $G$ is the gain of the radar antenna, $\sigma$ is the radar cross section of the target, $A_{e}$ is the effective area of the radar antenna, $\mathrm{t}_{\mathrm{ot}}$ is the time the radar antenna is pointed at the target, and $\mathrm{R}$ is the range to the target. Every radar has a minimum signal energy that it can detect, $S_{\min }$. This minimum signal energy determines the maximum range $\left(R_{\max }\right)$ at which the radar can detect a given target.

$$
R_{\max } \cong \sqrt[4]{\frac{P_{a v g} G \sigma A_{e} t_{o t}}{\left(4 \pi^{2}\right) S_{\min }}}
$$

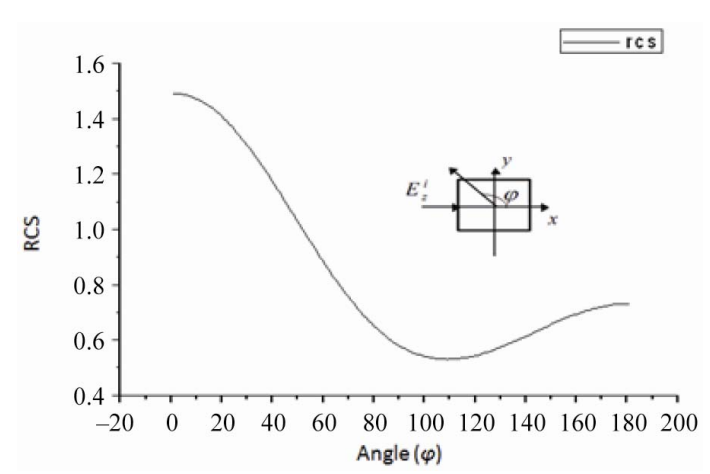

Figure 5. RCS behaviour with respect to the azimuth angle.
According to Equation (7), the distance at which a target can be detected for given radar configuration varies with the fourth root of its RCS. Figure 6 gives some understanding of just how little radar power is typically reflected back from the target and received by the radar. In this case, the target presents the same aspect to the radar at ranges from 1 to 50 miles. At a range of 50 miles, the relative power received by the radar is only $1.6 \times$ $10^{-5} \%$ of the strength at one mile. This diagram graphically illustrates how significant the effect of energy dissipation is with distance, and how sensitive radars must be to detect targets at even short ranges. Hence the cost associated to radar risk is based on a UAV's exposure to enemy radar. In this work, the cost of radar risk involves the amount of energy received by enemy radar at several locations based on the distance between the UAV and the radar. We assume that the UAV's radar signature is uniform in all directions and is proportional to $1 / R^{4}$ (where $R$ is the distance between the UAV and the enemy radar); the UAVs are assigned simplified flight dynamics and performance constraints in two-dimensions; and all tracking radars are given simplified detection properties. Based on the assumptions stated above, we classify the navigational space into different levels of risk as described in the following chapter. The next step is to compute a stealthy path, steering the UAV clear and around known radar locations. The generated path should carry minimal radar risks, and adhere to physical and motion constraints. Also, the distance between the UAV and the radars should be maximized in order to minimize the energy received at the radar.

\section{Problem Formulation}

To describe the problem, consider the contour based navigational space shown in Figure 7. The objective is to find a path which minimizes the UAV's exposure to radar sites (small triangles) from the current UAV position (circle) to the target location (star). In the present study, it is assumed that the hostile radar zones are

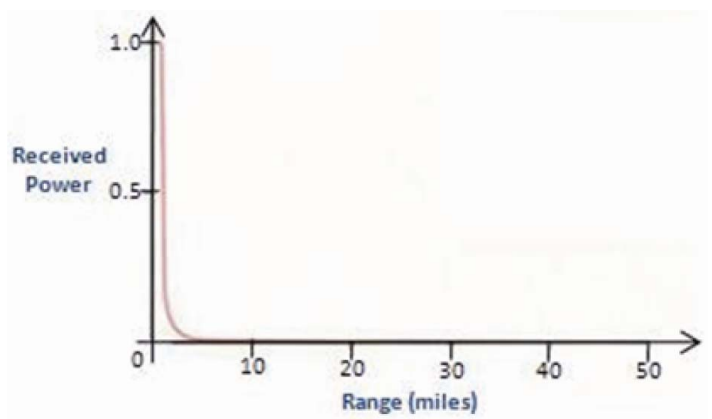

Figure 6. Reduction in the strength of target echoes with range. 


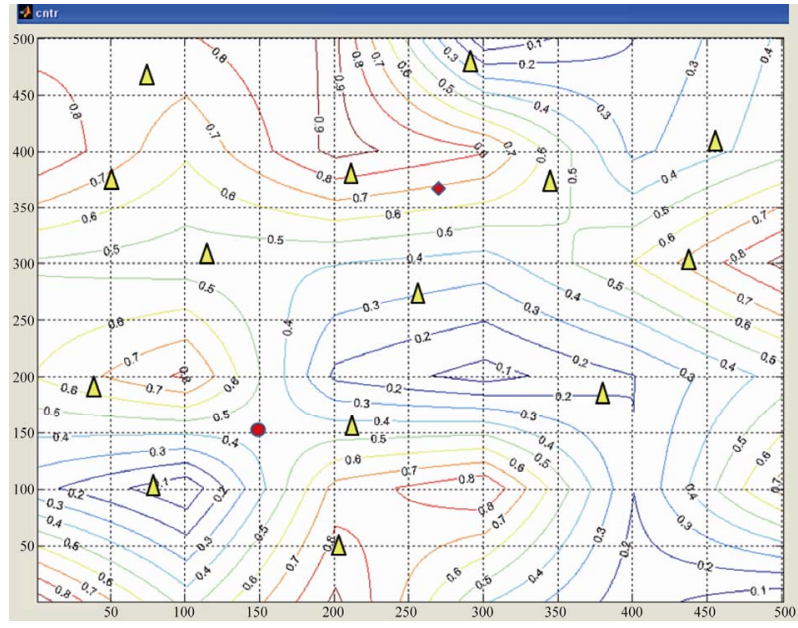

Figure 7. Contour based navigational space with known radar sites.

known beforehand. The contours are used to denote elevation and depth on maps. From these contours, a sense of the general terrain can be determined. The details of the radar zone can be acquired using satellite data or from surveillance data. The start point and the end point of the flight are known and it is required to find a feasible path connecting these two points. The threshold altitude value is determined by the user which assumes knowledge of the enemy terrain. By flying at a userspecified threshold altitude value, the aircraft can take advantage of terrain masking and avoid detection by enemy radars. The aircraft works by transmitting a radar signal towards the ground area and the signal returns can then be analysed to see how the terrain ahead varies, which can then be used by the aircraft's autopilot to specify the threshold altitude value. The specified threshold allows the aircraft to fly at reasonably constant height above the earth so as to avoid the radar detection. In this work, the path of the UAV is smoothened using cubic Bsplines, which is controlled by manipulating a number of control points. The generated path should carry minimal radar risks, and adhere to physical and motion constraints.

Given a single radar located at the origin and a single aircraft travelling from initial point to the destination, Pachter [22] showed that an objective function for minimizing cost, is

$$
J=\int_{0}^{\frac{l}{v}} \frac{1}{R^{4}(t)} \mathrm{d} t
$$

where $v$ is the constant speed of the aircraft and $l$ is the path length. A closed form solution to this problem was obtained using the calculus of variations, with the limitation that the aircraft traverses an angle with respect to the radar of less than $60^{\circ}$. Beyond this limit, the optimal path length is infinite but the cost remains finite. The objective cost function Equation (8), is augmented to include the rest of the radar at the navigational space, giving

$$
J=\int_{0}^{\frac{l}{v}} \sum_{i=1}^{m} \frac{\alpha_{i}}{R^{4}(t)} \mathrm{d} t
$$

The cost associated with radar risk involves the amount of energy $\alpha_{i}$, received by enemy radar based on the distance from the UAV to the radar. We first look for appropriate waypoints that the UAV should traverse in planning a path which minimizes the radar exposure.

\section{Generation of Nodes}

The procedure starts by requesting from the user the detection altitude or maximum elevation desired which assumes knowledge of the enemy terrain. Nodes below the detection altitude are extracted and used as base in the generation of straight line segments. The nodes above the detection altitude are discarded, given that at those elevations the UAVs could be detected. Appropriate value for the parameter is based on the user's knowledge of: the terrain; the operation of the UAVs; the purpose, logistics, and safety of the mission. This means that the user's input is a determinant in the quality of the resulting path. As shown in Figure 8, the initial nodes can be searched by specifying the detection altitude for the UAV. The next step is to make use of heuristic search algorithm to compute a stealthy path, steering the UAV clear and around known radar locations.

\section{Implementation}

\subsection{Generation of Nodes}

This search algorithm works by starting at UAV's pre-

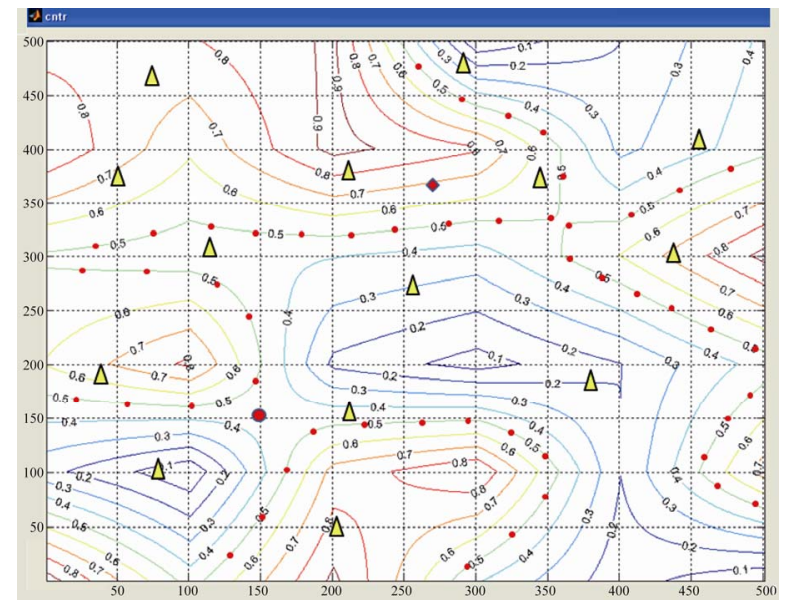

Figure 8. Generated waypoints based on user-specified threshold altitude value. 
sent position (red circle). It adds the heuristic function value and determines the expansion order of nodes. We make use of a distance-plus-cost heuristic function, $f(n)$ to determine the order in which the search visits nodes in the space. We assume that the UAV's radar signature is uniform in all directions and is proportional to $1 / R^{4}$ where $R$ is the aircraft range. We modify the evaluation function by adding the cost associated with radar risk to the $f(n)$ as follows:

$$
f(n)=w \times g(n)+(1-w) \times h(n)+\sum_{i=0}^{m} \frac{c_{i}}{R_{i}^{4}}
$$

where $w$ is a value in the range $[0,1] ; g(n)$ is the pathcost function which shows the intensity between the current node and node $n ; h(n)$ is the distance value which is estimated from node $n$ to the goal node; $c_{i}$ is relative to $R_{i}$ and $m$ is the number of enemy radars located at the navigational space. The $h(n)$ plays a significant role in the search process when $w$ value is smaller; the number of vertices to be explored would be decreased. The choice of $w$ between 0 and 1 gives the flexibility to place weight on exposure to threats or fuel expenditure depending on the particular mission scenario. For example when $w=0$, the search process would proceed from the starting location to the target location by exploring the least number of vertices. However the generated path is not stealthy and may cause UAV to enter high radar risk region. A $w$ value closer to 0 would result in the shortest paths, with little regard for the exposure to enemy radar, while a value closer to 1 would result in paths that avoid threat exposure at the expense of longer path length. For this case, the cost weighting parameter $w$ was chosen to give a reasonable tradeoff between proximity of the path to threats and the path length. The evaluation function, (10) is tested empirically and compared in terms of the number of nodes visited. During the test, the cost weighting parameter $w$ is varied from 0.0 to 1.0 and the test result is demonstrated in Table 1. The experimentally defined value for the $w$ ranges from $[0.1,0.3]$ in which the generated path is optimal and computationally inexpensive, which is in line with our algorithm objectives. The generated path is neither the safest possible path nor the shortest possible path, but represents a compromise between the two objectives.

The algorithm traverses various paths from the starting location to the goal. At the same time, it compares the risk cost of all the vertices. Starting with the initial node, it maintains a priority queue of nodes to be traversed, known as the openQueue (see Listing 1). The lower $f(n)$ for a given node $n$, the higher its priority. At each step of the algorithm, the node with the lowest $f(n)$ value is removed from the queue, the $f$ and $h$ values of its neighbors
Table 1. Test results.

\begin{tabular}{ccc}
\hline $\begin{array}{c}\text { Cost Weighting Pa- } \\
\text { rameter, } w\end{array}$ & Explored Nodes & $\begin{array}{c}\text { Cost of the Generated } \\
\text { Path }\end{array}$ \\
\hline$w$ is not used & 2405 & 103 \\
0 & 385 & 117.5 \\
0.1 & 369 & 112.5 \\
0.2 & 365 & 110.5 \\
0.3 & 365 & 106.5 \\
0.4 & 468 & 104 \\
0.5 & 2405 & 103 \\
0.6 & 4975 & 101 \\
0.7 & 7073 & 100.5 \\
0.8 & 7521 & 99 \\
0.9 & 7766 & 97 \\
1.0 & 8085 & 97 \\
\hline
\end{tabular}

The algorithm traverses various paths from the starting location to the goal. At the same time, it compares the risk cost of all the vertices. Starting with the initial node, it maintains a priority queue of nodes to be traversed, known as the openQueue (see Listing 1). The lower $f(n)$ for a given node $n$, the higher its priority. At each step of the algorithm, the node with the lowest $f(n)$ value is removed from the queue, the $f$ and $h$ values of its neighbors are updated accordingly, and these neighbors with the least radar cost are added to the openQueue. The conditions of adding a node to the openQueue are as follows:

- The node is not in high radar risk region;

- The node is movable and does not exist in the openQueue;

- The distance between the starting point and the current node is shorter than the earlier estimated distance;

- The distance between the starting point and the current node does not violate the pre-specified detection altitude.

If the four conditions are satisfied, then the current node will be added to the openQueue. The algorithm continues until a goal node has a lower $f$ value than any node in the queue. If the actual optimal path is desired, the algorithm may also update each neighbor with its immediate predecessor in the best path found so far; this information can then be used to reconstruct the path by working backwards from the goal node. The output of this stage is a set of straight line segments connecting a subset of the nodes resulting from the waypoint generation process, as long as the link does not violate the pre-specified detection altitude. The path planning algorithm is implemented in a MATLAB environment and a sample of the result can be seen in Figure 9.

The pseudo-code for the algorithm is as shown in Listing 1.

The next step is to modify the generated path by using a series of cubic splines to optimize the path by moving away from high radar risks and improving the navigability of the path for the UAV. 


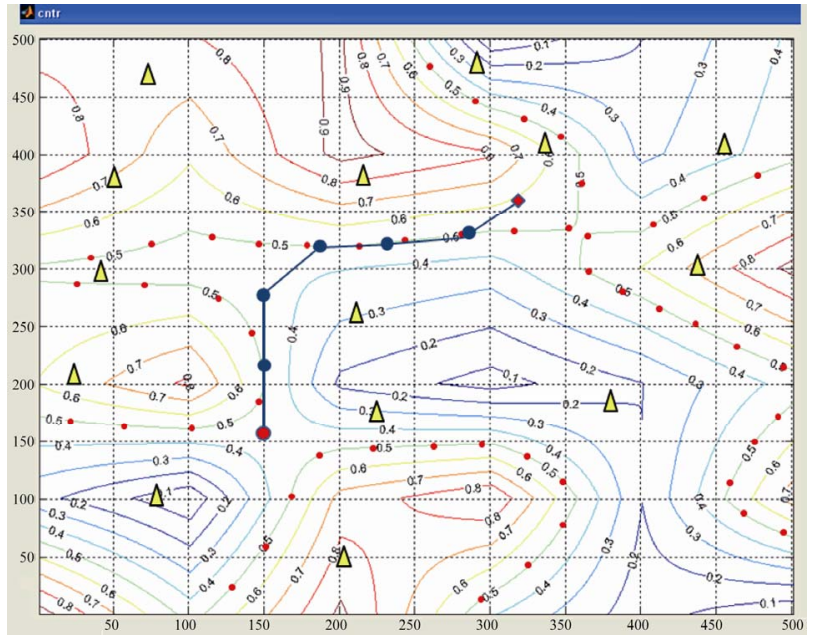

Figure 9. Generated path with least radar risk based on user-specified detection altitude.

\subsection{Trajectory Generation}

As a first step, the path is parameterized in both $x$ and $y$ directions. An initial spline knot is fixed at the UAV starting location, while the final spline knot is fixed at the first edge of the path. We begin by splitting each edge of the path into three segments, each one described by a different B-spline curve [23]. In this work, we calculate the radar risk cost at several locations along each edge and take the length of the edge into account. The radar risk cost was calculated at three points along each edge: $L_{i} / 6, L_{i} / 2$, and $5 L_{i} / 6$, where $L_{i}$ is the length of edge $i$. The radar risk cost for each edge may be calculated by

$$
J_{e}=L_{i} \sum_{j=1}^{N} \sum_{i=1}^{M}\left(\frac{1}{d_{1 / 6, i, j}^{4}}+\frac{1}{d_{1 / 2, i, j}^{4}}+\frac{1}{d_{5 / 6, i, j}^{4}}\right) \alpha_{j}
$$

where $N$ is the number of enemy radars; $M$ is the number of discrete point and $d_{1 / 6, i, j}$ is the distance from the $1 / 6^{\text {th }}$ point on the $i^{\text {th }}$ edge to the $j^{\text {th }}$ enemy radar. The goal now is to find the $(x, y)$ locations which minimize the exposure to the enemy radar for the middle knots. We apply the graph search algorithm to look for the middle knots with minimal risk cost for each edge of the path. We determine the interpolating curve based on tangent vectors and curvature vectors for each pair of points. The interpolation problem is solved by assuming $p=3$, which produces the $C^{2}$ cubic spline [24]. The parameters $\hat{u}_{k}$ are used to determine the knot vector $u$ as

$$
\begin{gathered}
u_{0}=u_{1}=u_{2}=\hat{u}_{0}, \quad u_{n+4}=u_{n+5}=u_{n+6}=\hat{u}_{n}, \\
u_{j+3}=\hat{u}_{j}, j=0, \cdots, n
\end{gathered}
$$

Since the number of control points, $m+1$, and the number of the knots, $n_{\text {knot }}+1$, are related by $n_{\text {knot }}=m+4$ and, as can be easily deduced from (12), $n_{\text {knot }}=n+6$, the
Function search_path (start, goal)

closedQueue: $=$ the empty queue/*The set of nodes already evaluated $* /$

openQueue: = queue containing the initial nodel

*The set of tentative nodes to be evaluated*/

$g[$ start $]:=0 / *$ Distance from start along optimal path*/

$h[$ start $]:=$ estimate_of_distance (start, goal)

$f[$ start $]:=h[$ start $]]^{*}$ Estimated total distance from start to goal through $y^{* /}$

while openQueue is not empty

$n:=$ the node in openQueue having the lowest $f[]$ value

if $n=$ goal

return reconstruct_path (came_from, goal)

remove $n$ from openQueue

add $n$ to closedQueue

for each y in neighbor_nodes $(n)$

if $y$ in closedQueue

continue

tentative_g: $=g[n]+$ dist_between $(n, y)+$ risk_cost

/*Compares the risk cost*/

tentative_is_better: $=$ false
if $y$ not in openQueue
add y to openQueue
$h[y]:=$ estimate_of_distance $(y$, goal $)$
tentative_is_better: $=$ true
elseif tentative_g $<[y]$
tentative_is_better: $=$ true
if tentative_is_better $=$ true
came_from $[y]:=n$
$g[y]:=$ tentative_g
$f[y]:=g[y]+h[y]$
return failure
if came_from[current_node $]$ is set $p=$ recon-
path $($ came_from, came_from $[$ current_node $])$
return $(p+$ current_node)
else
return the empty path

Listing 1. Pseudo-code for the path planning algorithm.

unknown variables $p_{j}$ are $n+3$. Once the control points $p_{j}, j=0, \cdots, n+2$ are known, and given the knot vector $u$, the B-spline is completely defined. Once this step has been completed, the procedure switches to the algorithm (as shown in Listing 2) to determine how to connect the knots. Note that some experimentally defined knots are added by the algorithm in order to smoothen the spline curve. 
Function spline_path (start, goal)

locate_points ()$/{ }^{*}$ get coordinates $(x, y)$ of middle knots*

$m \_$points: $=$the vector list ${ }^{*}$ to store control points*/

$m$ nodes: = the vector list $/ *$ to store small nodes $* /$

$m_{-}$steps: $=$number of steps $/{ }^{*}$ steps for spline $* /$

add points $(x, y) /{ }^{*}$ store $(x, y)$ in a vector list*/

while $(x, y)$ is not goal

new_controlpoint_added ()

/*spline curve based on the control points and nodes*/

for int $i=0$ to $m \_$steps

compute blending function

$\boldsymbol{S}_{i}(t)=\left[\begin{array}{llll}t^{3} & t^{2} & t & 1\end{array}\right] \frac{1}{6}\left[\begin{array}{cccc}-1 & 3 & -3 & 1 \\ 3 & -6 & 3 & 0 \\ -3 & 0 & 3 & 0 \\ 1 & 4 & 1 & 0\end{array}\right]\left[\begin{array}{c}p_{i-1} \\ p_{i} \\ p_{i+1} \\ p_{i+2}\end{array}\right]$

for $t \in[0,1]$

get_point (double u, const GLVector \& P0, const GLVector \& P1, const GLVector \& P2, const GLVector \& P3)

/*return coordinate $(x, y)$ for particular $t^{*} /$ add_node (const GLVector \& node)/*add small nodes in between the control points and we make use of 100 small nodes in this work*/

end for

end while

render_spline ()$/ *$ display generated curve*/

return the spline path

Listing 2. Pseudo-code for generating a spline path.

It is worth noticing that the interpolation by means of cubic B-splines guarantees the $C^{2}$ continuity of the geometric path. Owing to the continuity of the curve derivatives, it is not possible to obtain a path with sharp corners that is not flyable by the UAVs. An illustration of the generated solution is shown in Figure 10.

\section{Conclusions and Future Works}

This paper presents a contour based path planner to generate an optimal path for UAVs over hostile terrain. The proposed method is flexible given that it allows the user to input a threshold altitude value to avoid radar detection and terrain collisions. The key strengths of the method are: 1) the ability to plot a safe path to the target while minimizing exposure to the enemy radar; 2) the generated paths are smoothened using cubic splines to

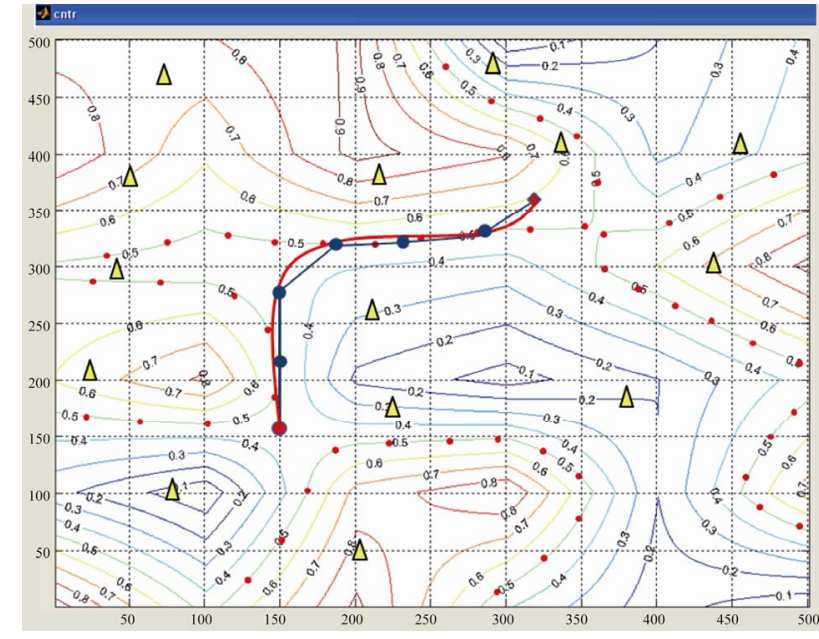

Figure 10. Optimal path based on heuristic search algorithm and user-specified threshold altitude.

improve navigability and 3) computational efficiency is achieved. Using this planning approach, the ability to generate feasible paths has been demonstrated through simulations. The information provided in this paper is significant for the planning of an air reconnaissance mission. However the procedure has limitations and is sensitive to some of the input parameters, leaving room for improvements in future research based on computational intelligence approaches [25-28].

\section{Acknowledgements}

The authors gratefully acknowledge the funding support from Temasek Defence Systems Institute, Singapore.

\section{References}

[1] A. Agarwal, M. H. Lim, M. J. Er and T. N. Nguyen, "Rectilinear Workspace Partitioning for Parallel Coverage Using Multiple UAVs," Advanced Robotics, Vol. 21, No. 1, 2007, pp. 105-120.

[2] K. K. Lim, Y. S. Ong, M. H. Lim and A. Agarwal, "Hybrid Ant Colony Algorithm for Path Planning in Sparse Graphs," Soft Computing Journal, Vol. 12, No. 10, 2008, pp. 981-994.

[3] C. W. Yeu, M. H. Lim, G. Huang, A. Agarwal and Y. S. Ong, "A New Machine Learning Paradigm for Terrain Reconstruction," IEEE Geoscience and Remote Sensing Letters, Vol. 3, No. 3, 2006, pp. 981-994. doi:10.1109/LGRS.2006.873687

[4] L. Davis, "Warp Speed: Path Planning for Star Trek: Armada," AAAI Spring Symposium, AAAI Press, Menlo Park, 2000.

[5] E. Frazzoli, M. Dahleh and E. Feron, "Real-Time Motion Planning for Agile Autonomous Vehicles," Journal of Guidance, Control and Dynamics, Vol. 25, No. 1, 2002, pp. 116-129. doi: $10.2514 / 2.4856$ 
[6] N. H. Sleumer and N. Tschichold-Gürman, "Exact Cell Decomposition of Arrangements Used for Path Planning in Robotics," Technical Reports 329, ETH Zürich, Institute of Theoretical Computer Science, 1999.

[7] J. C. Latombe, "Robot Motion Planning," Kulwer Academic Publishers, Boston, 1991.

[8] T. Lozano-Pyrez and M. A. Wesley, "An Algorithm for Planning Collision-Free Paths among Polyhedral Obstacles," Communications of the ACM, Vo1. 22, No. 10, 1979, pp. 565-570.

[9] M. Jun, "Path Planning for Unmanned Aerial Vehicles in Uncertain and Adversarial Environments," In: S. Butenko, R. Murphey and P. Pardalos, Eds., Cooperative Control: Models, Applicarions and Algorithms, Kluwer, 2003, pp. 95-111.

[10] J. Hilgert, K. Hirsch, T. Bertram and M. Hiller, "Emergency Path Planning for AutonomousVehicles Using Elastic Band Theory," Advanced Intelligent Mechatronics, Vol. 2, 2003, pp. 1390-1395.

[11] T. Sattel and T. Brandt, "Ground Vehicle Guidance Along Collision-Free Trajectories Using Elastic Bands," Proceedings of 2005 American Control Conference, Portla, 2005, pp. 4991-4999.

[12] J. Hwang, R. C. Arkin and D. Kwon, "Mobile Robots at Your Fingertip: Bezier Curve On-Line Trajectory Generation for Supervisory Control," Proceedings of Intelligent Robots and Systems (IROS 2003), Las Vegas, Vol. 2, 2003, pp. 1444-1449.

[13] J. Aleotti, S. Caselli and G. Maccherozzi, "Trajectory Reconstruction with NURBS Curves for Robot Programming by Demonstration," Proceedings of Computational Intelligence in Robotics and Automation, Barcelona, 2005, pp. 73-78.

[14] K. B. Judd and T. W. McLain, "Spline Based Path Planning for Unmanned Air Vehicles," AIAA Guidance, Navigation, and Control Conference and Exhibit, Montreal, 2001.

[15] E. P. Anderson, R. W. Beard, and T. W. McLain, "RealTime Dynamic Trajectory Smoothing for Unmanned Air Vehicles," IEEE Transactions on Control Systems Technology, Vol. 13, No. 3, 2005, pp. 471-477. doi:10.1109/TCST.2004.839555

[16] E. M. Kan, M. H. Lim, S. P. Yeo, S. H. Shao and J. S. Ho, "Radar Aware Path Planning for UAVs," IEEE Symposium on Intelligent Systems and Applications, Trabzon, June 2009.
[17] E. M. Kan, S. P. Yeo, C. S.Tan, S. H. Shao and J. S. Ho, "Stealth Path Planning for UAVs in Hostile Radar Zones," Proceedings of the $11^{\text {th }}$ IASTED International Conference on Control and Applications, Cambridge, July 2009, pp. 54-60.

[18] F. W. Moore, "Radar Cross-Section Reduction via Route Planning and Intelligent Control," IEEE Transactions on Control Systems Technology, Vol. 10, No. 5, 2002, pp. 696-700. doi:10.1109/TCST.2002.801879

[19] U. F. Knott, "Radar Cross Section Measurements," Van Nostrand Reinhold, New York, 1993.

[20] L. Sevgi, "Complex Electromagnetic Problems and Numerical Simulation Approaches," IEEE Press/John Wiley \& Sons, New York, 2003.

[21] E. F. Knott, J. F. Shaeffer, and M. T. Tuley, "Radar Cross Section," 2nd Edition, Artech House, Norwood, 1993, p. 231.

[22] M. Pachter, D. R. Jacques and J. M. Hebert, "Minimizing Radar Exposure in Air Vehicle Path Planning," Proceedings of the 41st Israel Annual Conference on Aerospace Sciences, Tel-Aviv, February 2001.

[23] M. G. Cox, "The Numerical Evaluation of B-Splines," IMA Journal of Applied Mathematics, Vol. 10, No. 2, 1972, pp. 134-149. doi:10.1093/imamat/10.2.134

[24] R. H. Bartels, J. C. Beatty and B. A. Barsky, "An Introduction to Splines for Use in Computer Graphics and Geometric Modeling," Morgan Kaufmann Publishers, Massachusetts, 1987.

[25] Q. Cao, M. H. Lim, J. H. Li, Y. S. Ong and W. L. Ng, “A Context Switchable Fuzzy Inference Chip," IEEE Transactions on Fuzzy Systems, Vol. 14, No. 4, 2006, pp. $552-$ 567. doi:10.1109/TFUZZ.2006.876735

[26] M. H. Lim and Y Takefuji, "Implementing Fuzzy RuleBased Systems on Silicon Chips," IEEE Expert, Vol. 5, No. 1, 1990, pp. 31-45. doi:10.1109/64.50855

[27] R. Meuth, M. H. Lim, Y. S. Ong and D. C. Wunsh, “A Proposition on Memes and Meta-Memes in Computing for Higher-Order Learning," Memetic Computing, Vol. 1, No. 2, 2009, pp. 85-100. doi:10.1007/s12293-009-0011-1

[28] M. H. Lim, Q. Cao, J. H. Li and W. L. Ng, "Evolvable Hardware Using Context Switchable Fuzzy Inference Processor," IEEE Proceedings: Computers and Digital Techniques, Vol. 151, No. 4, 2004, pp. 301-311. doi:10.1049/ip-cdt:20040666 